\title{
Registro de ocorrência de duas espécies de potamotrigonídeos na região do Alto Rio Paraná e algumas considerações sobre sua biologia
}

\author{
Domingos Garrone Neto ${ }^{1,4}$, Vidal Haddad Jr. ${ }^{2}$, Maria José Alencar Vilela ${ }^{3}$ \& Virgínia Sanches Uieda \\ Biota Neotropica $v 7(n 1)$ \\ http://www.biotaneotropica.org.br/v7n1/pt/abstract?short-communication+bn00707012007 \\ Recebido em 21/06/06 \\ Versão reformulada recebida 01/10/06 \\ Publicado em 01/01/2007
${ }^{1}$ Departamento de Zoologia, Instituto de Biociências, Universidade Estadual Paulista - UNESP, CP 510, CEP 18618-000, Botucatu, SP, Brasil
e-mail: garroneneto@yahoo.com,vsuieda@ibb.unesp.br
${ }^{2}$ Departamento de Dermatologia e Radioterapia, Faculdade de Medicina, Universidade Estadual Paulista - UNESP, CP 557, CEP 18618-000, Botucatu, SP, Brasil
e-mail: haddadjr@fmb.unesp.br
${ }^{3}$ Departamento de Ciências Naturais, Universidade Federal de Mato Grosso do Sul - UFMS,
Avenida Ranulpho Marques Leal, 3484, CP 210, CEP 79600-030, Três Lagoas, MS, Brasil
e-mail:mjvilela@ceul.ufms.br
${ }^{4}$ Autor para correspondência: Domingos Garrone Neto, e-mail: garroneneto@yahoo.com

\begin{abstract}
Garrone Neto, D., Haddad Jr., V., Vilela, M.J.A. \& Uieda, V.S. Occurrence record of two potamotrigonids species in the Upper Paraná River and some aspects about their biology. Biota Neotrop. Jan/Apr 2007 vol. 7, no. 1 http://www.biotaneotropica.org.br/v7n1/pt/abstract?short-communication+bn00707012007 ISSN 1676-0603.

The freshwater stingrays of the Potamotrygonidae family are elasmobranchs restricted to the major river systems of South America. In Brazil, the occurrence of potamotrigonids is known in all the Amazonian Basin, some rivers of the Northeastern region and in the Paraguay-Paraná Basin. The upper course of the Paraná River, separated in the past from the medium and low courses by Sete-Quedas Waterfalls, today is connected to these areas due to the dams constructed in great part of its course. The dams had destroyed natural barriers, making possible the dispersion of many species of aquatic organisms to the headwaters of the Paraná River. We report the occurrence record of two species of stingrays in the Upper Paraná River, which were not part of native fauna about two decades ago.

Keywords: geographic distribution, biological invasions, freshwater stingrays, Potamotrygonidae, Paraná River.

\section{Resumo}

Garrone Neto, D., Haddad Jr., V., Vilela, M.J.A. \& Uieda, V.S. Registro de ocorrência de duas espécies de potamotrigonídeos na região do Alto Rio Paraná e algumas considerações sobre sua biologia. Biota Neotrop. Biota Neotrop. Jan/Apr 2007 vol. 7, no. 1 http://www.biotaneotropica.org.br/v7n1/pt/abstract?shortcommunication+bn00707012007 ISSN 1676-0603.

A distribuição geográfica das raias da família Potamotrygonidae é restrita aos principais sistemas fluviais da América do Sul. No Brasil, a ocorrência de potamotrigonídeos é conhecida em toda a Bacia Amazônica, em alguns rios da região Nordeste e na Bacia Paraguai-Paraná. O alto curso do Rio Paraná, separado no passado dos trechos médio e baixo pelas Cachoeiras de Sete-Quedas, hoje está conectado a essas áreas devido ao represamento de grande parte de seu curso. A construção das barragens destruiu obstáculos naturais, possibilitando a dispersão de diversas espécies de organismos aquáticos rumo ao alto curso do Rio Paraná. Reportamos aqui o registro da ocorrência de duas espécies de raias no Alto Rio Paraná, as quais não faziam parte da fauna nativa da região há pouco mais de duas décadas atrás.
\end{abstract}

Palavras-chave: distribuição geográfica, invasões biológicas, raias de água doce, Potamotrygonidae, Rio Paraná. 


\section{Introdução}

A família Potamotrygonidae (Elasmobranchii: Myliobatiformes) compreende um grupo de condríctes que possuem adaptações para a vida exclusiva em água doce (Thorson et al. 1978). A distribuição geográfica das 16 a 20 espécies válidas até o momento é restrita aos principais sistemas fluviais da América do Sul, incluindo rios da Venezuela, Guiana, Suriname, Guiana Francesa, Colômbia, Peru, Bolívia, Paraguai, Argentina, Uruguai e Brasil (Achenbach \& Achenbach 1976, Rosa 1985, Carvalho et al. 2003). Neste último país, é na Bacia Amazônica onde o maior número de espécies é encontrado (cerca de 13), excetuando-se as espécies em descrição e/ou ainda não descritas (Charvet-Almeida et al. 2002). O Nordeste do Brasil apresenta registros pontuais, nos Estados do Maranhão e Piauí (Rosa 1985). Nas regiões Centro-Oeste e Sudeste, a Bacia Paraguai-Paraná abriga cerca de sete espécies, embora esse número possa variar devido à possibilidade de existirem espécies sinônimas e/ou outras ainda não descritas (Rosa 1985, Carvalho et al. 2003).

No Rio Paraná, até o final dos anos 70, a ocorrência de raias era assinalada apenas para os trechos situados à jusante das Sete-Quedas, uma importante barreira geográfica situada no Município de Guaíra, Estado do Paraná (Vilela et al. 2004). Com o fim do enchimento do reservatório da Usina Hidrelétrica de Itaipu no ano de 1982, esse obstáculo natural, que isolou por muito tempo a maioria da ictiofauna do Alto Rio Paraná da fauna remanescente dos sistemas à jusante, foi destruído, possibilitando a colonização do trecho situado à montante de Guaíra por novas espécies. Algumas dessas espécies, como o tucunaré (Cichla ocellaris) e a corvina (Plagioscion squamosissimus), passaram a desempenhar o papel de espécies invasoras, provocando impactos negativos sobre a fauna aquática nativa (Brasil 1998, Vilela et al. 2004). Outras, como as raias (Potamotrygon spp.), se dispersaram rapidamente e se envolveram em acidentes com seres humanos, despertando interesse pelos possíveis impactos gerados sobre a população ribeirinha e turistas.

No presente estudo, registramos a ocorrência de Potamotrygon falkneri Castex \& Maciel (1963) e Potamotrygon motoro (Müller \& Henle 1841) na região do Alto Rio Paraná, ampliando a distribuição geográfica da família Potamotrygonidae, e apontamos algumas considerações sobre a biologia dessas espécies.

\section{Materiais e Métodos}

O Rio Paraná nasce da confluência dos rios Grande e Paranaíba, a aproximadamente $20^{\circ}$ de latitude sul e $51^{\circ}$ de longitude oeste. Desde sua formação, tem largura superior a $1 \mathrm{~km}$ e vazão mínima de mais de $1000 \mathrm{~m}^{3} / \mathrm{s}$. Partindo da confluência dos rios Grande e Paranaíba, o Rio Paraná corre em território brasileiro com orientação geral sudeste por cerca de $619 \mathrm{~km}$ até atingir as Cachoeiras de Sete-Quedas ou Quedas de Guaíra, inundadas pelo reservatório de Itaipu nos anos 1980 (Souza 2000). Desse ponto, inflete para sul, passando a fazer fronteira entre Brasil e Paraguai numa extensão de $190 \mathrm{~km}$ até a foz do Rio Iguaçu, a partir de onde passa a ser limite entre a Argentina e o Paraguai (Vazzoler et al. 1997, Souza 2000).

A região do Alto Rio Paraná compreende todo o trecho situado acima das Sete-Quedas de Guaíra - PR, abrigando tributários importantes como os rios Grande, Paranaíba, Tietê, Paranapanema, Verde e Sucuriú (Souza 2000). A bacia de drenagem do Alto Rio Paraná possui aproximadamente $900.000 \mathrm{~km}^{2}$, incluindo a região norte-noroeste do Estado do Paraná, o sul-sudeste de Mato Grosso do Sul, a porção ocidental do Estado de São Paulo, o sudoeste de Minas Gerais, o sul de Goiás e uma pequena área do Paraguai oriental adjacente a Mato Grosso do Sul (Vazzoler et al. 1997).

Entre 2002 e 2005 foram coletados 104 exemplares de raias da família Potamotrygonidae nos rios Paraná, Tietê, Paranapanema e Su- curiú, com o auxílio de puçás, arpões e anzóis armados em espinhéis. Para tal, foram realizadas dez viagens científicas, sendo uma na foz do Rio Paranapanema, uma na foz do Rio Tietê e oito na região da Usina Hidrelétrica Engenheiro Souza Dias - UHE Jupiá (Município de Castilho, Estado de São Paulo), no Rio Paraná. As coletas foram realizadas predominantemente durante o dia, embarcadas, com o emprego de técnicas de mergulho livre e pesca artesanal, e contaram com o apoio de pescadores das colônias locais. Os exemplares capturados foram identificados seguindo a chave de identificação elaborada por Rosa (1985) e também com o auxílio de especialistas (Ricardo Rosa e Patrícia Charvet-Almeida-UFPB), e depositados na coleção ictiológica do Laboratório de Biologia e Genética de Peixes da Universidade Estadual Paulista-UNESP, Campus Botucatu.

Para todos os exemplares coletados foram obtidos o peso (em quilogramas) e as medidas, em milímetros, referentes ao comprimento total, comprimento do corpo, comprimento da cauda e largura do disco. A dieta dos animais capturados foi determinada através da análise do conteúdo estomacal sob estereomicroscópio, segundo Hyslop (1980).

\section{Resultados e Discussão}

Modificações ambientais por obras de engenharia são reconhecidamente importantes facilitadores de invasões biológicas em todo o mundo (Bertness 1984, Vitousek et al. 1996). No Rio Paraná, a perda de barreiras naturais pela construção de usinas hidrelétricas favoreceu a colonização do Alto Rio Paraná por diversas espécies exóticas - incluindo raias, que vem aproveitando as eclusas e escadas de peixes existentes nas barragens para ampliar sua área de distribuição (Garrone Neto, observações pessoais). Estudos realizados na região demonstraram que muitas espécies exóticas se estabeleceram, em maior ou menor grau (Vilela et al. 2004). As raias são alguns desses representantes e hoje, após coletas realizadas nas proximidades da UHE Jupiá, na foz do Rio Tietê e nos baixos Paranapanema e Sucuriú, sua área de distribuição foi conhecida na região do Alto Rio Paraná (Figura 1).

Potamotrygon falkneri e Potamotrygon motoro foram as espécies de raias capturadas na área de estudo (Figuras 2 e 3). Potamotrygon falkneri foi registrada no Rio Paraná, próximo aos municípios de Castilho no Estado de São Paulo e Três Lagoas no Estado de Mato Grosso do Sul (2047' 19' S e 51 37' 22”'W), e na região da foz do Rio Tietê (2039' 17' S e 51 $30^{\circ}$ ' 40" W), no Estado de São Paulo. O tamanho máximo observado para a espécie foi de $52 \mathrm{~cm}$ de largura de disco, contra $47 \mathrm{~cm}$ referidos na literatura (Carvalho et al. 2003). Os estudos em desenvolvimento sobre a reprodução desta espécie sugerem que as fêmeas parem seus filhotes durante a estação das chuvas, já que somente entre os meses de dezembro e janeiro, fêmeas capturadas abortaram fetos em estágio avançado de desenvolvimento (Garrone Neto, observações pessoais). A análise da dieta de exemplares capturados nas proximidades da UHE Jupiá mostrou notória diversidade de itens, incluindo moluscos, crustáceos, pequenos peixes (inclusive descartes oriundos das pescas artesanal e esportiva) e insetos aquáticos (estes em grande quantidade).

Potamotrygon motoro foi registrada nos mesmos locais que P. falkneri e também na região da foz dos rios Paranapanema $\left(22^{\circ} 35^{\prime} 39^{\prime}\right.$ ' S e $\left.51^{\circ} 56^{\prime} 41^{\prime \prime} \mathrm{W}\right)$ e Sucuriú $\left(20^{\circ} 40^{\prime} 04^{\prime}\right.$ ' S e $51^{\circ} 44^{\prime} 24^{\prime}$ 'W). A ocorrência de $P$. motoro foi assinalada para o Rio Paranapanema por Britto (2004), mas espécimes-testemunho não foram depositados em coleções. O tamanho máximo observado para esta espécie foi de $47 \mathrm{~cm}$ de largura de disco. Sua dieta, analisada em exemplares capturados próximos a Três Lagoas, mostrou-se muito semelhante à de $P$. falkneri.

As duas espécies aparentemente ocupam o mesmo habitat e apresentam hábitos predominantemente bentônicos, sendo encontradas 


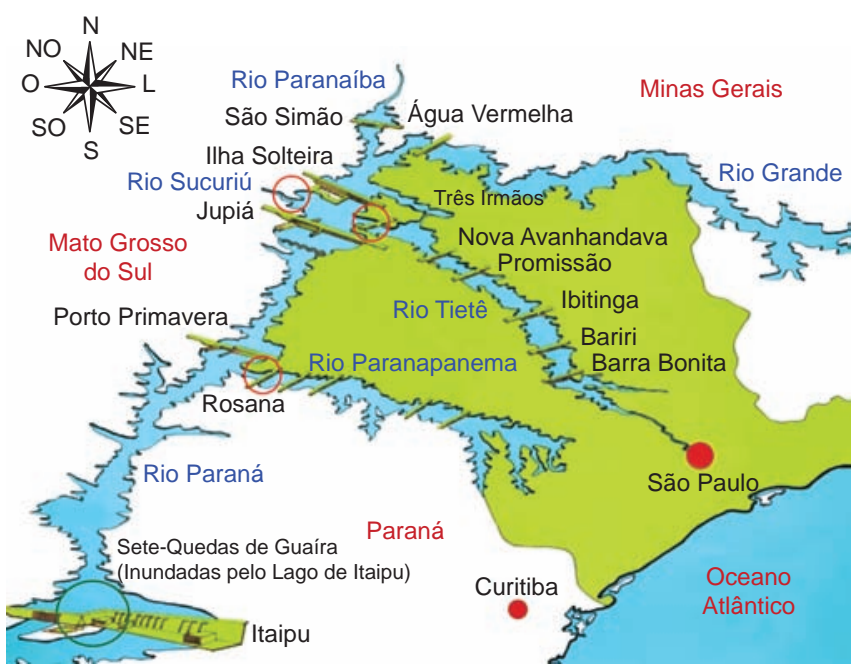

Figura 1. Mapa da região do Alto Rio Paraná, com a indicação dos principais rios e usinas hidrelétricas. Círculos em vermelho indicam os novos locais de ocorrência das raias; o círculo verde indica o provável local onde a mais recente dispersão do grupo ocorreu.

(Modificado de http://www.estanciabarrabonita.com.br).

Figure 1. Map of the Upper Paraná River region, with the indication of the main rivers and dams. Red circles indicate the new occurrence places of the rays; green circle indicate the probable place where the most recent dispersion of the group occurred.

(Modified from http://www.estanciabarrabonita.com.br)

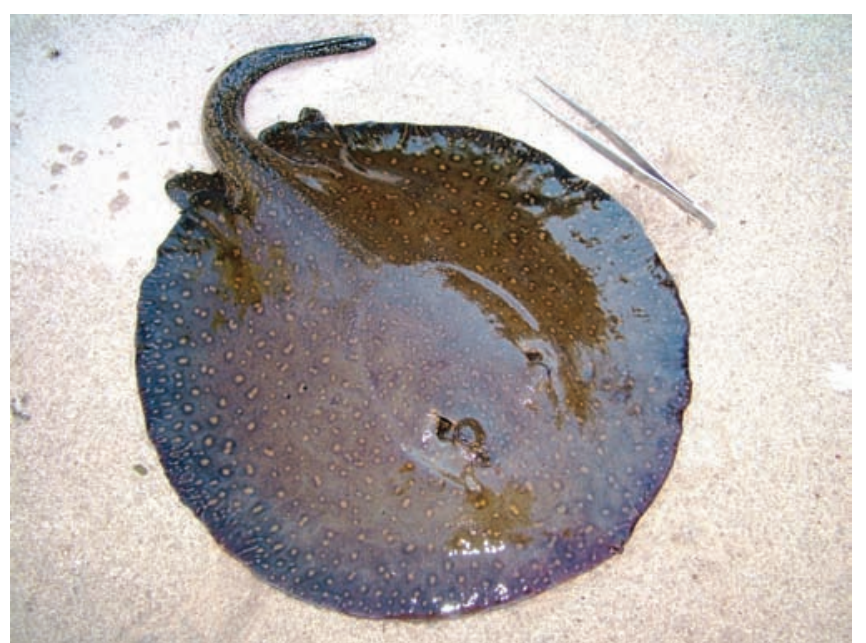

Figura 2. Fêmea de Potamotrygon falkneri Castex \& Maciel (1963). Peso: 0,660 kg. Largura do disco: $310 \mathrm{~mm}$.

Figure 2. Potamotrygon falkneri Castex \& Maciel (1963) female. Weight: $0.660 \mathrm{~kg}$. Disc width: $310 \mathrm{~mm}$.

preferencialmente em locais entre dois e quatro metros de profundidade, com velocidade de corrente e substrato bastante variáveis. Os exemplares capturados apresentaram policromatismo acentuado, tanto em relação à classe de tamanho, quanto em função do micro-habitat ocupado. Estudos preliminares de taxonomia verificaram diferenças morfológicas entre alguns indivíduos, o que, aliado a grande variação no padrão de coloração das espécies estudadas, dificultou a identificação de alguns exemplares. Esse fato sugeriu, inclusive, a possibilidade da existência de híbridos e até mesmo de espécies ainda não descritas.

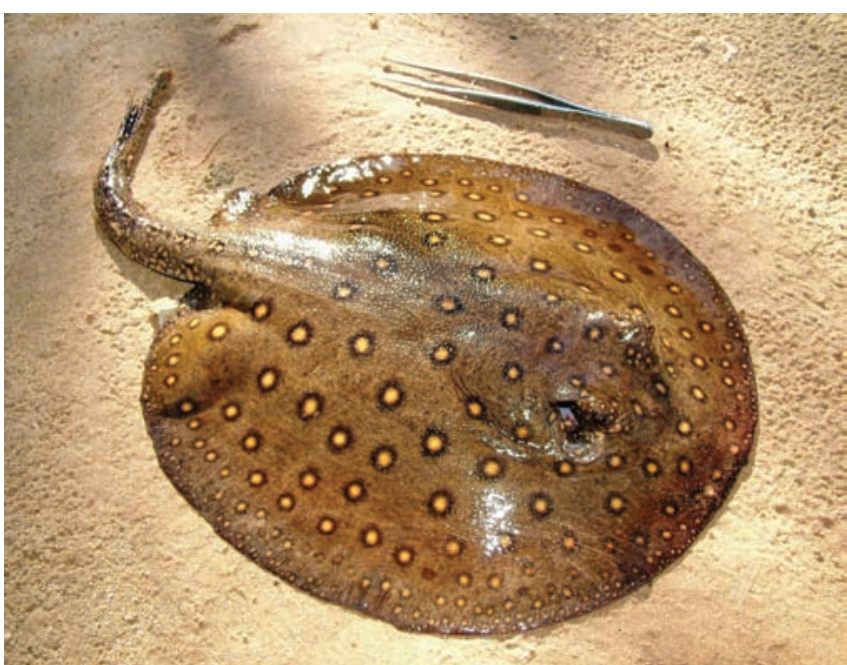

Figura 3. Fêmea de Potamotrygon motoro (Müller \& Henle 1841) Peso: 0,510 kg. Largura do disco: $280 \mathrm{~mm}$.

Figure 3. Potamotrygon motoro (Müller \& Henle 1841) female. Weight: $0.510 \mathrm{~kg}$. Disc width: $280 \mathrm{~mm}$.

Com o intuito de investigar essas hipóteses, estudos taxonômicos com o uso de ferramentas moleculares estão em andamento.

Apesar das dúvidas quanto à identificação de alguns exemplares capturados, está claro que populações de $P$. falkneri e $P$. motoro se estabeleceram na região do Alto Rio Paraná e que sua distribuição geográfica vem se ampliando a cada ano, favorecida pelas eclusas existentes nas usinas hidrelétricas instaladas ao longo da drenagem Tietê-Paraná. Embora não sejam animais habitualmente agressivos, causando acidentes apenas quando pisoteadas ou manuseadas de maneira indevida, as raias são dotadas de ferrões que podem provocar ferimentos dolorosos, de difícil cicatrização (Haddad Jr. et al. 2004). Injúrias desse tipo são comumente observadas entre habitantes das regiões norte e centro-oeste do Brasil, onde sua elevada morbidade é reconhecida como um importante problema de saúde pública (Haddad Jr. et al. 2004, Garrone Neto et al. 2005). Em locais onde as raias não faziam parte da fauna aquática nativa, como o alto curso do Rio Paraná e áreas adjacentes, vale ressaltar que o desconhecimento da população acerca desses animais poderá favorecer a ocorrência de acidentes. Nesse contexto, ressalta-se a importância de informações ecológicas e naturalísticas como base para a realização de estudos epidemiológicos e atividades educativas, com a finalidade de esclarecer a população humana sobre o risco do contato com animais potencialmente perigosos à saúde.

\section{Agradecimentos}

Agradecemos a Sandro Geraldo de Castro Britto e Mateus Ferrareze Feitosa pelas informações complementares acerca da ocorrência de raias no Baixo Paranapanema, a Thiago Buosi Silva, Ottilie Carolina Forster e Cléia Natália Pascoalim Caíres pelo apoio nos trabalhos de campo, a Ricardo Rosa e Patrícia Charvet-Almeida pela colaboração na identificação das espécies de raias coletadas, e aos dois assessores anônimos pelas valiosas sugestões. Domingos Garrone Neto é bolsista de doutorado do Conselho Nacional do Desenvolvimento Científico e Tecnológico (CNPq) e teve apoio financeiro da Coordenação de Aperfeiçoamento de Pessoal de Nível Superior (CAPES), por intermédio da coordenação do curso de PósGraduação em Ciências Biológicas - A/C Zoologia da Universidade Estadual Paulista-UNESP, Campus Botucatu. 


\section{Referências Bibliográficas}

ACHENBACH, G.M. \& ACHENBACH, S.V.M. 1976. Notas acerca de algumas espécies de "raya fluvial" (Batoidei, Potamotrygonidae) que frecuentan el sistema hidrográfico del rio Paraná médio en el departamento La Capital (Santa Fé - Argentina). Com. Mus. Prov. Cs. Nat. 8:1-34.

BERTNESS, M.D. 1984. Habitat and community modification by an introduced herbivorous snail. Ecology 65:370-381.

BRASIL. 1998. Portaria n $^{\circ} 145 / 98$, de 29 de outubro de 1998: dispõe sobre introduções, re-introduções e transferências de espécies aquáticas no Brasil. Diário Oficial da União, Brasília.

BRITTO, S.G.C. 2004. Peixes do Rio Paranapanema. $1^{\mathrm{a}}$ ed. Horizonte Geográfico, São Paulo.

CARVALHO, M.R., LOVEJOY, N.R. \& ROSA, R.S. 2003. Family Potamotrygonidae (river stingrays). In Check List of the Freshwater Fishes of South and Central America (R.E. REIS, S.O. KULLANDER \& C.J. FERRARIS JR., orgs.). Edipucrs, Porto Alegre, p.22-28.

CHARVET-ALMEIDA, P., ARAÚJO, M.L.G., ROSA, R.S. \& RINCÓN, G. 2002. Neotropical freshwater stingrays: diversity and conservation status. Shark News 14:47-51.

GARRONE NETO, D., CORDEIRO, R.C \& HADDAD JR., V. 2005. Acidentes do trabalho em pescadores artesanais da região do Médio Rio Araguaia, TO, Brasil. Cad. Saúde Púb. 21(3):795-803.

HADDAD JR., V., GARRONE NETO, D., PAULA NETO, J.B., MARQUES, F.P.L. \& BARBARO, K.C. 2004. Freshwater stingrays: study of epidemiologic, clinic and therapeutic aspects and some enzymatic activies of the venom based on 84 envenomings in humans. Toxicon 48:287-294.

HYSLOP, E.J. 1980. Stomach contents analysis - a review of methods and their application. J. Fish Biol. 17:411-429.

ROSA, R.S. 1985. A systematic revision of the South American freshwater stingrays (Chondrichthyes: Potamotrygonidae). $\mathrm{PhD}$ thesis, The College of William and Mary, Williamsburg.

SOUZA, I.A. 2000. Geografia física do Rio Paraná, Brasil. Dissertação de Mestrado, Instituto de Geociências, Universidade Estadual de Londrina, Londrina.
THORSON, T.B., WOOTON, R.M. \& GEORGI, T.D. 1978. Rectal gland of freshwater stingrays, Potamotrygon spp. (Chondrichthyes: Potamotrygonidae). Biol. Bull. 154:508-516.

VAZZOLER, A.E.A.M., AGOSTINHO, A.A. \& HAHN, N.S. 1997. A Planície de inundação do Alto Rio Paraná: aspectos físicos, biológicos e sócioeconômicos. Editora da Universidade Estadual de Maringá, Maringá.

VILELA, M.J.A., FRANCO, R.A.M. \& ALMEIDA, N.V.A. 2004. Monitoramento da pesca no reservatório de Porto Primavera (UHE Eng ${ }^{\circ}$ Sérgio Motta), no Rio Paraná. Relatório Final (Agosto/2000-Novembro/2003), CESP/FAPEC/UFMS, Três Lagoas.

VITOUSEK, P.M., D’ANTONIO, C.M., LOOPE, L.L., WESTBROOKS, R. 1996. Biological invasions as global environmental change. Am. Scient. $84: 468-478$

Titulo: Registro de ocorrência de duas espécies de potamotrigonídeos na região do Alto Rio Paraná e algumas considerações sobre sua biologia.

Autores: Garrone Neto, D, Haddad Jr., V, Vilela, MJA e Uieda, VS

Biota Neotropica, Vol.7 (número 1): 2007 http://www.biotaneotropica.org.br/v7n1/pt/abstract?shortcommunication+bn00707012007

Recebido em 21/06/06 - Versão reformulada recebida 01/10/06 - Publicado em 01/01/2007

ISSN 1676-0603 